\title{
Clinical Manifestations and Maternal and Infant Outcomes of Pregnancy Complicated with Thrombocytopenia
}

\author{
Limin Hou, Qiuying Gao, Ben Niu, Yanchuan $\mathrm{Li}^{*}$ \\ Shaanxi Provincial People's Hospital, Xi'an 710068, Shaanxi Province, China \\ *Corresponding author: Yanchuan Li, hlm3582696@163.com
}

\begin{abstract}
Objective: To explore the clinical manifestations as well as maternal and infant outcomes of pregnancy complicated with thrombocytopenia. Methods: A total of 478 pregnant women with thrombocytopenia, treated in Shaanxi Provincial People's Hospital from July 2019 to June 2021, were selected as the research group, while 200 normal pregnant women, treated during the same period, were selected as the control group to analyze gestational thrombocytopenia (GT), idiopathic thrombocytopenic purpura (ITP), pregnancy-induced hypertension (PIH), adverse maternal and infant outcomes, etc. Results: Among the 478 patients in the research group, the main causes of pregnancy complicated with thrombocytopenia were GT, ITP, and PIH, accounting for $75.51 \%, 6.49 \%$, and $8.79 \%$, respectively, while other causes accounted for $9.21 \%$. There was no significant difference between the research group and the control group in the amount of intrapartum bleeding, premature delivery, stillbirth, thrombocytopenia, and neonatal asphyxia, but there was significant difference in the mode of delivery $(P$ $<0.05)$. Conclusion: The traditional indexes of coagulation function are not abnormal with decreased platelets in pregnant women with thrombocytopenia, but the indexes of thromboelasticity are abnormal and can reflect the changes of coagulation function. The cesarean section rate of patients with thrombocytopenia in pregnancy increases with the decrease of platelet count, but the mode of delivery should be determined in consideration of specific conditions.
\end{abstract}

Keywords: Pregnancy; Thrombocytopenia; Maternal and infant outcome

Publication date: November 2021; Online publication: November 30, 2021

\section{Introduction}

Platelets are an important component of blood. After the proliferation, differentiation, development, and maturation of hematopoietic stem cells in the bone marrow, platelets are released into the blood to play a hemostatic role. When thrombocytopenia occurs, it may cause skin and mucosal bleeding; in severe cases, visceral bleeding can even occur ${ }^{[1]}$. Thrombocytopenia refers to a platelet count of less than $150 \times 10^{9} / \mathrm{L}$ and its pathogenesis involves decreased platelet production and/or increased consumption, which can be seen in leukemia, aplastic anemia (AA), lymphoma, ITP, bone marrow suppression after chemotherapy, hypersplenism, etc. ${ }^{[2]}$. Patients with thrombocytopenia in pregnancy have bleeding tendencies, with skin and mucosal bleeding being more common. In severe cases, visceral bleeding, vaginal bleeding, and even intracranial bleeding may endanger one's life, resulting in adverse pregnancy outcomes. In addition to affecting the mother, the condition can also cause premature delivery, abortion, neonatal arrhythmias, thrombocytopenia, intracranial hemorrhage, and other risks threatening the life of newborns. Different causes and degrees of thrombocytopenia in pregnancy may lead to differences in maternal and fetal outcomes, so early detection, identification, and appropriate interventions are needed. There have been many analyses on the etiology, treatment, and prognosis of thrombocytopenia in pregnancy abroad, but 
there is no unified diagnosis and treatment guidelines for this condition in China yet. Pregnancy complicated with thrombocytopenia is not only a common disease in obstetrics and gynecology, but also a type of disease with high frequency in hematology consultation in obstetrics and gynecology. Therefore, analyzing the characteristics of thrombocytopenia in pregnancy is of great significance to improve the understanding of the disease.

\section{Data and methods}

\subsection{General information}

A total of 478 pregnant women with thrombocytopenia, treated in Shaanxi Provincial People's Hospital from July 2019 to June 2021, were selected as the research group, while 200 normal pregnant women, treated during the same period, were selected as the control group. The age of the patients in the research group ranged from 18 years old to 39 years old, with an average age of $29.12 \pm 1.33$. The age of the normal pregnant women in the control group during the same period was from 19 years old to 41 years old, with an average age of $28.91 \pm 1.23$. There was no significant difference in the general data between the two groups.

The inclusion criteria were patients who filed at Shaanxi Provincial People's Hospital and were on regular prenatal examinations; patients who did not consume any thrombocytopenic drugs within the past two weeks; patients who were consistent with pregnancy complicated with thrombocytopenia; patients and their families who agreed to the study.

The exclusion criteria were patients with congenital deficiency of coagulation factors; patients with plasma fibrinogen related diseases; patients with incomplete clinical data.

\subsection{Methods}

The clinical indexes of all patients were collected before delivery, including platelet count, four components of the coagulation test and thromboelasticity test. The delivery mode, amount of intrapartum bleeding, and occurrence of prematurity, stillbirth, thrombocytopenia, asphyxia, and other uncertain delivery outcomes were also noted.

\subsection{Observation indicators}

Gestational thrombocytopenia (GT), primary immune thrombocytopenia (ITP), pregnancy-induced hypertension $(\mathrm{PIH})$, adverse maternal and infant outcomes, etc.

\subsection{Statistical analysis}

SPSS 23.0 statistical software was used. If the measurement data comply with normal distribution and homogeneity of variance, the mean \pm the standard deviation is expressed by variance test and LSD test is used for intragroup comparison. If it does not conform to the normality or homogeneity of variance, it is expressed by the median and upper and lower quartiles, and the comparison is performed by nonparametric test. The counting data were expressed by constituent ratio. Chi square test or Fisher's exact probability test was used for comparison. $P<0.05$ indicates that the difference is statistically significant.

\section{Results}

\subsection{Etiological distribution of pregnancy complicated with thrombocytopenia}

Among the 478 patients in the research group, the main causes of pregnancy complicated with thrombocytopenia were GT, ITP, and PIH, accounting for $75.51 \%, 6.49 \%$, and $8.79 \%$, respectively. Other 
causes accounted for $9.21 \%$. GT, ITP, and PIH accounted for the causes of pregnancy complicated with thrombocytopenia (Table 1).

Table 1. Etiological distribution of pregnancy complicated with thrombocytopenia (n/\%)

\begin{tabular}{ccccc}
\hline Group & GT & ITP & PIH & Others \\
\hline Research group $(\mathrm{n}=478)$ & $361(75.51)$ & $31(6.49)$ & $42(8.79)$ & $44(9.21)$ \\
\hline
\end{tabular}

\subsection{Maternal and infant outcomes}

In the study, there was no significant difference between the research group and the control group in the amount of intrapartum bleeding, premature delivery, stillbirth, thrombocytopenia, and neonatal asphyxia, but there was significant difference in the mode of delivery $(P<0.05)$ (Table 2).

Table 2. Comparison of maternal and infant outcomes

\begin{tabular}{|c|c|c|c|c|c|c|c|}
\hline \multirow[t]{2}{*}{ Group } & \multicolumn{2}{|c|}{ Mode of delivery $(\mathrm{n} / \%)$} & \multirow{2}{*}{$\begin{array}{c}\text { Intrapartum } \\
\text { bleeding } \\
(\overline{\mathrm{x}} \pm \mathrm{s}, \mathrm{mL})\end{array}$} & \multirow{2}{*}{$\begin{array}{c}\text { Premature } \\
\text { delivery } \\
(\mathrm{n} / \%)\end{array}$} & \multirow{2}{*}{$\begin{array}{l}\text { Stillbirth } \\
(\mathrm{n} / \%)\end{array}$} & \multirow{2}{*}{$\begin{array}{l}\text { Thrombocytopenia } \\
(\mathrm{n} / \%)\end{array}$} & \multirow{2}{*}{$\begin{array}{c}\text { Neonatal } \\
\text { asphyxia } \\
(\mathrm{n} / \%)\end{array}$} \\
\hline & $\begin{array}{l}\text { Vaginal } \\
\text { delivery }\end{array}$ & $\begin{array}{c}\text { Delivery by } \\
\text { cesarean } \\
\text { section }\end{array}$ & & & & & \\
\hline $\begin{array}{l}\text { Research group } \\
(\mathrm{n}=478)\end{array}$ & $\begin{array}{c}369 \\
(77.20)\end{array}$ & $109(22.80)$ & $289 \pm 47.88$ & $36(7.53)$ & $0(0.00)$ & $25(5.23)$ & $6(1.26)$ \\
\hline $\begin{array}{l}\text { Control group } \\
(\mathrm{n}=200)\end{array}$ & $\begin{array}{c}178 \\
(89.00)\end{array}$ & $22(11.00)$ & $284 \pm 46.99$ & $8(4.00)$ & $0(0.00)$ & $8(4.00)$ & $0(0.00)$ \\
\hline $\mathrm{X}^{2} / \mathrm{T}$ & \multicolumn{2}{|c|}{12.6020} & 1.2468 & 2.8976 & - & 0.4608 & - \\
\hline $\mathrm{P}$ & \multicolumn{2}{|c|}{$<0.05$} & 0.2129 & 0.0887 & - & 0.4973 & - \\
\hline
\end{tabular}

\section{Discussion}

Thrombocytopenia is a common blood disorder in pregnant women. With the increase of gestational weeks, maternal blood volume continues to increase and at the same time, platelet consumption in the blood circulation increases. There are changes to the quantity and quality of platelets in the body, in which this change is a physiological change. The degree of platelet decline in terms of its quantity is low, which has minimal impact on the delivery mode as well as maternal and infant outcome ${ }^{[4]}$. On the contrary, pathological thrombocytopenia often leads to adverse maternal and infant outcomes, including premature delivery, massive intrapartum or postpartum hemorrhage, neonatal thrombocytopenia, and so on.

Thrombocytopenia internationally refers to a platelet count of less than $150 \times 10^{9} / \mathrm{L}$, but the general default platelet count of less than $100 \times 10^{9} / \mathrm{L}$ is significant ${ }^{[5]}$. Pregnancy complicated with thrombocytopenia ranks second in hematological diseases during pregnancy ${ }^{[6]}$. There are various causes of pregnancy complicated with thrombocytopenia, which can be divided into pregnancy specific and nonpregnancy specific. Pregnancy specific includes pregnancy-associated thrombocytopenia (PAT), hypertensive disorders complicating pregnancy (HDCP), acute fatty liver of pregnancy (AFLP), etc. while for non-pregnancy specific, ITP is common in addition to systemic lupus erythematosus (SLE), AA, Ma, myelodysplastic syndrome (MDS), antiphospholipid syndrome (APS), thrombotic thrombocytopenic purpura (TTP), etc., involving the blood system, immune system, and other systems in the whole body. Among all causes, PAT accounts for the highest proportion, about $70-80 \%{ }^{[6]}$, followed by HDCP of about $15 \%{ }^{[2]}$; ITP accounts for about $3 \%{ }^{[3]}$, while other causes are relatively rare. There are differences in the 
pathogenesis in regard to the causes of thrombocytopenia in pregnancy. PAT mainly involves an increase in blood volume and hemodilution during pregnancy, placental circulation, as well as other effects ${ }^{[6]}$. Pregnancy complicated with ITP involves humoral and cellular immune disorders, changes in estrogen level, desialylation, and abnormal regulatory B cell function. HDCP activates the coagulation system due to increased blood pressure and vasospasm, resulting in increased platelet utilization. Blood system diseases include AA, MDS, bone marrow abnormalities, and thrombocytopenia. In immune diseases such as SLE and APS, the formation of autoimmune antibodies and the increase in peripheral platelet destruction are the main pathogenies. The three most common etiological types are consistent with the literatures at home and abroad. The proportions of ITP and HDCP are slightly different. The possible reason is that in ITP patients, the clinical manifestations and degree of thrombocytopenia are relatively obvious; thus, the condition can be diagnosed earlier. In addition, since Shaanxi Provincial People's Hospital is a comprehensive third-class hospital with strong strength in various departments, patients with ITP in pregnancy have a higher probability of referral from subordinate hospitals to this hospital. On the other hand, HDCP has an insidious onset. Patients with HDCP are often diagnosed and treated in local hospitals, so the proportion is low.

In conclusion, the traditional coagulation indexes for pregnancy with thrombocytopenia are not abnormal with the decrease in platelets, but the detection indexes of thromboelasticity are abnormal and can reflect the changes to the coagulation function. The cesarean section rate of patients with thrombocytopenia in pregnancy increases with the decrease of platelet count, but the mode of delivery should be determined in consideration of specific conditions.

\section{Disclosure statement}

The authors declare that there is no conflict of interest.

\section{References}

[1] Dou D, Zhang H, Feng Y, et al., 2021, Maternal and Fetal Prognosis of Cesarean Section with Different Anesthesia Methods Due to Thrombocytopenia Caused by Pregnancy Complicated with Blood Diseases: A Single Center 12-Year Retrospective Study. Journal of Practical Medicine, 37(13): 17191722.

[2] Cheng X, Zhang J, 2021, Application Effect of Evidence-Based Nursing in Pregnancy Patients with Thrombocytopenia. Thrombosis and Hemostasis, 27(03): 535-536.

[3] Rong Y, 2021, Clinical Observation of Immunoglobulin in the Treatment of Late Pregnancy Complicated with Thrombocytopenia. Journal of Henan Medical College, 33(01): 43-45.

[4] Zhou L, Zhang X, 2021, Etiological Analysis of Pregnancy Complicated with Thrombocytopenia and Its Impact on Delivery Mode and Maternal and Infant Outcome. China Maternal and Child Health Care, 36(03): 499-502.

[5] Tong T, Yin F, Liu R, 2021, Etiology of Pregnancy Complicated with Thrombocytopenia and Its Impact on Maternal and Fetal Outcomes. Chinese Journal of Reproductive Health, 32(01): 11-15, 20.

[6] Gao G, Jin Y, Wang J, et al., 2021, Discussion on the Etiology, Treatment Scheme and Delivery Mode of Pregnancy Complicated with Thrombocytopenia. Armed Police Medicine, 31(11): 940-943. 